\title{
Commentaries
}

\section{Life, death, and varices}

In patients with cirrhosis and portal hypertension, gastrointestinal haemorrhage is a major complication and cause of death. Oesophageal varices are present in approximately $50 \%$ of patients with cirrhosis; the prevalence is higher in Child-Pugh $\mathrm{C}$ patients than in those with ChildPugh A-B. Among patients with varices, the risk of gastrointestinal haemorrhage ranges from $30 \%$ to $50 \%$ and half of these patients die within six weeks after bleeding. Thus $10-15 \%$ of unselected patients with cirrhosis die from gastrointestinal bleeding. The number of deaths has decreased in the last few decades as a result of modern techniques (see below). The exact mechanisms of variceal rupture have not yet been determined and thus no ideal treatment has been found. However, haemorrhage has been shown to cause different complications such as sepsis or renal failure which may be responsible for death. In patients admitted for variceal haemorrhage, certain prognostic factors for death have been determined such as age, Child-Pugh C, hepatocellular carcinoma, early rebleeding, hepatic encephalopathy, and renal failure. ${ }^{1}$

Ideally, patients admitted for acute variceal haemorrhage must be taken to a medical centre with an emergency facility and an intensive care unit with experience in variceal bleeders. In the last 20 years, various medical, surgical, radiological, pharmacological, and endoscopic treatments have been used and most are effective in controlling variceal haemorrhage compared with no treatment or placebo. Most of these treatments, except endoscopic therapy, act by decreasing portal pressure. There is a negative relationship between treatment efficacy and invasiveness of the method. For example, surgical shunts or transjugular intrahepatic portosystemic stent shunts are more effective than pharmacological and endoscopic treatments but are more invasive and thus associated with more side effects. Although all of these treatments stop variceal haemorrhage, a significant difference in mortality has only been found with endoscopic sclerotherapy or terlipressin administration compared with placebo or no treatment. The discrepancy between the efficacy of treatment on variceal bleeding and survival rate suggests that other factors play a role in survival. Emergency endoscopic sclerotherapy is now the gold standard in the management of acute variceal haemorrhage as it increases hospital survival. ${ }^{2}$ A recent meta-analysis showed however that endoscopic sclerotherapy and variceal ligation are equally effective, suggesting that variceal ligation may also improve survival rate with fewer complications. ${ }^{3}$ Two controlled studies have shown that terlipressin administration is effective both in controlling bleeding and improving survival rate. In one trial, terlipressin stopped bleeding in $90 \%$ of patients with a significant difference in survival rate before discharge. ${ }^{4}$ In a second study, terlipressin was administered with glyceryl trinitrate by an intensive care team, within one hour after an emergency call before admission. ${ }^{5}$ In this trial, mortality due to bleeding episodes was significantly lower in the terlipressin group than in the placebo group at day 15 and day 42 . These findings suggest that this drug should be administered as soon as possible before endoscopic investigation. Moreover, it is interesting to note that these two treatments (sclerotherapy and terlipressin administration) are equally effective in the initial control of variceal bleeding and in the prevention of early rebleeding. ${ }^{6}$

The second type of therapy is for the prevention of the complications of haemorrhage. Patients with gastrointestinal bleeding are at a high risk of bacterial infection and thus antibiotics must be prescribed. A meta-analysis confirmed that the use of prophylactic antibiotics prevents bacterial infection but also the risk of early rebleeding and more important, significantly improves survival rate. ${ }^{7}$ Prevention of liver failure, including encephalopathy and renal failure, may also play a major role in the improvement in survival rate. Administration of blood products must be limited as it has been suggested that excess blood products may induce early rebleeding. Similarly, limiting certain more or less invasive investigations may reduce severe complications. Accordingly, improvements in survival rate may depend in part on the type of treatment. This is relatively clear for terlipressin administration ${ }^{8}$ but no explanation exists for endoscopic treatment.

Finally, mortality due to acute variceal haemorrhage has significantly decreased in the last few decades. Preliminary results of one study showed that inhospital mortality has decreased by $50 \%$ over the past 15 years. ${ }^{9}$ Moreover, the authors showed that although there were no deaths in Child-Pugh A patients following gastrointestinal bleeding, $25 \%$ of Child-Pugh C patients still died from haemorrhage. A second recent study followed 5000 patients in two cohorts separated by an interval of 11 years, who were followed for more than six years. ${ }^{10}$ The authors demonstrated that mortality in the late cohort significantly declined at 30 days (approximately 30\%) and at six years (approximately $6 \%$ ). These results were despite the fact that patients in the late cohort had more severe liver disease. Thus improvement in survival rate is even more significant with multivariate analysis. This study also showed that patients treated with sclerotherapy during the first hospitalisation had a lower mortality rate than the rest of the late cohort. In this issue of Gut, McCormick and O'Keefe have calculated the survival rate in patients with cirrhosis admitted for a first episode of variceal haemorrhage (see page 682). ${ }^{11}$ They compared the survival rate from "control" groups in approximately 1500 patients from 1960 to 2000. The results show a significant reduction (approximately 40\%) in bleeding related mortality.

Although this improved survival rate after variceal haemorrhage might be expected, variceal haemorrhage was controlled for more than 20 years without any significant effects on survival rate. The only treatments known to improve survival rate are pharmacological or endoscopic treatments and antibiotic administration (see above). Nevertheless, a certain number of patients still die of variceal haemorrhage, suggesting that other factors must be studied. Thus new ideas, hypotheses, and approaches are needed to further our understanding of the treatment and mechanisms of variceal haemorrhage.

D LEBREC

Laboratoire d'Hémodynamique Splanchnique et de Biologie Vasculaire,

INSERM U-481 and Service d'Hépatologie,

Hôpital Beaujon, 92118 Clichy, France

lebrec@bichat.inserm.fr 
1 Del Olmo JA, Pena A, Serra MA, et al. Predictors of morbidity and mortality after the first episode of upper gastrointestinal bleeding in liver cirrhosis. $\mathcal{F}$ after the first episode of

2 Hartigan PM, Gebhard RL, Gregory PB. Sclerotherapy for actively bleeding esophageal varices in male alcoholics with cirrhosis. Gastrointest Endosc 1997;46:1-7.

3 deFranchis R, Primignani M. Endoscopic treatment for portal hypertension. Semin Liver Dis 1999;19:439-55.

4 Söderlund C, Magnusson I, Torngren S, et al. Terlipressin (triglycyl-lysine vasopressin) controls acute bleeding oesophageal varices. A double-blind, randomized, placebo-controlled trial. Scand F Gastroenterol 1990;25:622-30.

5 Levacher S, Letoumelin P, Pateron D, et al. Early administration of terlipressin plus glyceryl-trinitrate to control active upper gastrointestinal bleeding in cirrhotic patients. Lancet 1995;346:865-8

6 Escorsell A, Ruiz Del Arbol L, Planas R, et al. Multicenter randomized controlled trial of terlipressin versus sclerotherapy in the treatment of acute variceal bleeding: the TEST study. Hepatology 2000;32: 471-6.

7 Bernard B, Grangé JD, Khac EN, et al. Antibiotic prophylaxis for the prevention of bacterial infections in cirrhotic patients with gastrointestinal bleeding: a meta-analysis. Hepatology 1999;29:1655-61.

8 Lebrec D. A discussion of how terlipressin limits mortality in cases of bleeding oesophageal varices. Eur f Gastroenterol Hepatol 1998;10:549-52.

9 Pauwels A, Fourdan O, Carbonel N, et al. La mortalité des hémorragies digestives des cirrhoses a été réduite de plus de moitié en 15 ans. Gastroenterol Clin Biol 1998;22:(suppl 2):A27.

10 El-Serag HB, Everhart JE. Improved survival after variceal hemorrhage over an 11-year period in the department of veterans affairs. Am $\mathcal{F}$ Gastroenterol 2000;95:3566-73.

11 McCormick PA, O'Keefe C. Improving prognosis following a first variceal haemorrhage over four decades. Gut 2001;49:682-5.

\section{Can endoscopic papillary balloon dilation really preserve sphincter of Oddi function?}

The technique of endoscopic balloon dilation of the major duodenal papilla with extraction of bile duct stones was first reported in $1982^{1}$ but an unacceptably high rate of acute pancreatitis following the procedure impeded its acceptance until further reports in $1994^{2}$ and $1995^{3}$ reported its safety. A number of randomised controlled trials have demonstrated its effectiveness in clearing the bile ducts of stones compared with endoscopic sphincterotomy ${ }^{4-8}$ with variable short term complication rates similar to sphincterotomy. Currently, this technique has not become popular worldwide, but for reasons that are not clear has been adopted much more readily in Japan and Korea than elsewhere.

Those who advocate this technique over sphincterotomy do so on the premise that: (1) it is less traumatic, (2) it is simpler to perform, (3) it is as effective, (4) it avoids the immediate complications, such as bleeding and perforation, (5) it is cheaper or at least equivalent cost, (6) it preserves sphincter of Oddi function, and (7) it avoids the long term complications.

Many of these apparent advantages have been questioned in terms of technical performance and short term outcome and now there is new evidence from the report by Yasuda $e t$ al in this issue of $G u t^{9}$ that the long term advantages may also be in doubt (see page 686).

The above selection criteria in favour of balloon dilation of the papilla have been criticised and are discussed below under the same categories.

(1) Forcible balloon dilation of the papilla well beyond its normal resting diameter may be just as traumatic or more traumatic than its incision by sphincterotomy and indeed, the description of the degree of trauma is "in the eye of the beholder". (2) The technique is not necessarily simpler to perform than sphincterotomy, especially as current over-the-guidewire hydrostatic balloons are not designed for this application and may be difficult to keep in position during the dilation process. (3) The overall effectiveness of clearing the bile ducts is statistically equivalent to sphincterotomy although this is at the expense of a far greater need for mechanical lithotripsy and a small proportion of patients requiring additional procedures to achieve clearance. Recent use of intraductal ultrasound has cast doubt on the completeness of ductal clearance after balloon dilation with a retained stone rate of up to $33 \%$. (4)
The immediate complication rates, particularly acute pancreatitis, seem to be equivalent to sphincterotomy ${ }^{4-8}$ although the disturbing occurrence of severe pancreatitis and even death after balloon dilation in one recent randomised study ${ }^{6}$ calls safety into question if patients with unsuspected sphincter of Oddi dysfunction are treated in this way. (5) In the absence of prospective comparisons of costs, it seems unlikely that balloon dilation is cheaper. Even if a disposable balloon and sphincterotome are equivalent costs, the increased use of mechanical lithotripsy and some need for secondary procedures will have an adverse impact on this. (6 and 7) Studies to date ${ }^{4}{ }^{10}$ have suggested recovery of sphincter function after balloon dilation within a few weeks of the procedure but the study by Yasuda et al in this issue of $G u t^{9}$ has extended manometric follow up to one year and shown some recovery but to significantly subnormal pressures compared with preprocedure values. Although the authors discussed the possibility that the degree of "injury" might be dependent on the size and number of stones after balloon dilation, they were unable to show any correlation because of the small numbers involved in each stone size category. The implication is that the trauma of extracting stones or fragments of a size close to or at the limit of the degree of dilation of the papilla induces further injury which may not be able to recover completely. The clinical significance of this is uncertain but may be reflected in differences in long term complications between balloon dilation and sphincterotomy. In their combined retrospective and prospective study of a larger number of patients, ${ }^{9}$ a significantly lower incidence of biliary complications for balloon dilation included recurrent bile duct stones, cholangitis, and cholecystitis. Individual complications were significant for cholangitis which however did not occur in post-balloon dilation patients but was present in $3.2 \%$ of postsphincterotomy patients, and cholecystitis occurred in $2 \%$ and $8.8 \%$ of patients, respectively. The numbers were too small to distinguish whether or not the presence of cholelithiasis had a significant effect on this. The incidence of recurrent bile duct stones was $10 \%$ and $14 \%$, respectively, with no significant difference. In the smaller prospective part of their study, there were no significant differences in biliary complications at one year between the balloon dilation and sphincterotomy groups, although the recurrent stone rate was only $5.7 \%$ and $8.6 \%$, respectively, and the cholecystitis rate $3.3 \%$ and $3.8 \%$, respectively, with no reported incidence of cholangitis. As in previous studies, prospectively randomised patients showed no differences in acute complications which averaged $7 \%, 80 \%$ of which were pancreatitis. The groups were comparable with respect to number of stones (1-16), diameter of the largest 
stones (4-24 mm), and diameter of the bile duct (6-30 $\mathrm{mm}$ ) but the use of mechanical lithotripsy was significantly higher in the balloon dilation group (17\% compared with $9 \%$ in sphincterotomy patients).

With this new additional long term evidence it seems likely that endoscopic balloon dilation of the papilla for the treatment of choledocholithiasis would be considered as an alternative to sphincterotomy in certain circumstances but not as a replacement for it on a routine basis. Many centres have already adopted the practice of using balloon dilation in patients with previous surgically altered anatomy where access to the papilla is from the distal side and also in patients where there may be an uncorrectable coagulopathy which might carry increased risks of post-sphincterotomy bleeding. The converse of this discussion is the question of whether endoscopic sphincterotomy, particularly in the young patient, is all that undesirable to consider avoidance. The arguments were discussed in a leading article by Tham and colleagues ${ }^{11}$ indicating the expected rates for effectiveness, acceptable short and long term morbidity, apparent lack of serious consequences following sphincterotomy, general high standard of training in these techniques, together with the growth in laparoscopic management of choledocholithiasis during cholecystectomy.

The possible unsubstantiated differences in patient populations with choledocholithiasis in different countries may account for some of the differences in early morbidity with balloon dilation between the Far East and the West. I would currently advocate caution in the use of endoscopic dilation of the papilla for routine treatment of choledocholithiasis, except for patients in special circumstances, as detailed above, and then only for those with stone diameters up to the size of the balloon being employed to avoid excessive use of mechanical lithotripsy which in itself may have some influence on the complication rate. Unquestionably, further long term follow up studies are required to substantiate the findings by Yasuda and colleagues $^{9}$ but for the present time, sphincterotomy, a tried and tested technique now well into its third decade, would still seem to be the standard endoscopic modality to be offered to most patients with choledocholithiasis.

D L CARR-LOCKE

Division of Gastroenterology, Brigham and Women's Hospital,

Boston, Massachusetts, USA

dcarrlocke@partners.org

1 Staritz M, Ewe K, Meyer zum Buschenfelde KH. Endoscopic papillary dilatation: An alternative to papillotomy? Dtsch Med Wochenschr 1982;107:8957 .

2 MacMathuna P, White P, Clarke E, et al. Endoscopic spincteroplasty: a novel and safe alternative to papillotomy in the management of bile duct stones. Gut 1994;35:127-9.

3 MacMathuna PM. Endoscopic balloon sphincteroplasty (papillary dilation) for bile duct stones: efficacy, safety, and follow-up in 100 patients. Gastrointest Endosc 1995;42:468-74.

4 Minami A, Nakatsu T, Uchida N, et al. Papillary dilation vs sphincterotomy in endoscopic removal of bile duct stones-a randomized trial with manometric function. Dig Dis Sci 1995;40:2550-4

5 Bergman JJGHM, Rauws EAJ, Fockens P, et al. Randomized trial of endoscopic balloon dilation versus endoscopic sphincterotomy for removal of bile duct stones. Lancet 1997;349:124-9.

6 DiSario JA, Freeman ML, Bjorkman DJ, et al. Endoscopic balloon dilation compared to sphincterotomy (EDES) for extraction of bile duct stones. Gastrointest Endos 1997;45:AB129.

7 Chen YK, Degusman L, Godil A, et al. Prospective randomized trial of endoscopic balloon dilation versus sphincterotomy for extraction of bile duct stones. Gastrointest Endosc 1998;47:AB111.

8 Ochi Y, Mukawa K, Kiyosawa K, et al. Comparing the treatment outcomes of endoscopic papillary dilation and endoscopic sphincterotomy for removal of bile duct stones. F Gastroenterol Hepatol 1999;14:90-6.

9 Yasuda I, Tomita E, Enya M, et al. Can endoscopic papillary balloon dilation really preserve sphincter of Oddi function? Gut 2001;49:686-91.

10 Sato H, Kodama T, Takaaki J, et al. Endoscopic papillary balloon dilatation may preserve sphincter of Oddi function after common bile duct stone management: evaluation from the viewpoint of endoscopic manometry. Gut 1997;41:541-4.

11 Tham TCK, Carr-Locke DL, Collins JSA. Endoscopic sphincterotomy in the young patient: is there cause for concern? Gut 1997;40:697-700. 